\title{
Group Key Management with Network Mobility
}

\author{
Wee Hock Desmond Ng, Zhili Sun, Haitham Cruickshank \\ Centre for Communication Systems Research \\ University of Surrey \\ United Kingdom \\ $\{$ W.Ng $\mid$ Z.Sun | H.Cruickshank\}@surrey.ac.uk
}

\begin{abstract}
Secure multicast communication is important for both wired and wireless applications. For groups with frequent join or depart requests, a distributed architecture that partitions the group members into several areas is preferred. Inside each area, scalable algorithms such as Logical Key Hierarchy (LKH) can be used to update the group key. However, these algorithms do not consider mobile members traveling in a Mobile Network as a whole. In this paper, we proposed two group key management schemes, which treat mobile members traveling in a Mobile Network as a whole. Both schemes try to reduce the communication costs when the Mobile Network moves in or out of the area. Simulation results show significant reduction in communications costs even for small number of mobile members in the Mobile Network.
\end{abstract}

Keywords-group key management; secure multicast; network mobility

\section{INTRODUCTION}

IP multicast enables efficient group communication by allowing the sender to transmit a single copy of data, with network elements such as routers and switches making copies as necessary for the receivers. This solves the scalability issues at the sender side and allows better utilization of network resources such as bandwidth and buffer space. IP multicast not just benefits wired network applications [2] such as pay-perview and multi-party games., wireless applications [3-5] such as group-oriented mobile commerce, distance education and intelligent transportation systems gain significant boost from it.

In order for IP multicast to scale to virtually any group size, it relies on a single group address to identify the set of recipients rather than explicitly listing them. However, this anonymous receiver model prevents the content provider from charging the members. The only way to provide controlled access to data is to encrypt the multicast communication with a group key and distribute this shared group key to all authorized members [6-18]. Generally, applications might need confidentiality, authentication, integrity and non-repudiation [6]. If membership is dynamic, this shared group key has to be updated and redistributed to all authorized members securely every time whenever there is a change in the membership in order to provide forward and backward secrecy. Forward secrecy means a departing member cannot obtain information about future group communication and backward secrecy means that a joining member cannot obtain information about past group communication. Even if there is no change in the group membership, the group key might need to be refreshed after it has been in use for a given amount of time to thwart cryptanalysis.

In a typical multicast key management scheme, a trusted entity, known as the group controller (GC), is responsible for generating, distributing and replacing the group key for a multicast session. The operation for changing the key whenever there is a change in group membership is known as rekeying and rekeying cost denotes the total number of unicast or multicast messages that needs to disseminate to the members during rekeying. In order to minimize the rekeying costs for large group, the GC makes use of auxiliary keys, known as key encryption keys (KEKs), to update the group key. One popular technique is to employ a hierarchy of keys [9-13], in which each member is being assigned a set of keys based on its location in the key tree.

When the number of group members becomes large, group key management can become a significant overhead and a potential system overhead [18]. Thus, scalable approaches towards group key management [19-20] are needed. These approaches achieve scalability by partitioning the group members into a number of smaller areas to distribute the processing workload. The partition of the group members into areas may be done on either a physical or logical basis.

In wireless networks, mobility adds another dimension of complexity to the design of key management scheme by allowing members not only to join or depart the group but also transfer between areas $[16,18,21,22]$.

In this paper, we propose two group key management schemes for rekeying mobile members in a Mobile Network. A Mobile Network is a leaf network, which includes one or more mobile routers used to provide connectivity to the Internet $[23$, 24]. Typical examples are passengers traveling in train using their laptop wireless LAN cards to connect to wireless LAN access points deployed in the train. Existing algorithms do not consider mobile members in a Mobile Network as a whole, therefore resulting in a waste of network resources and computation power at both the members and GC side when a Mobile Network moves in or out of the area. In addition, outof-sync problem between the multicast data and the keys [25] might occur because the interval between each joining members might be very small when a Mobile Network moves into an area. It is also very inefficient since the first set of new keys is actually not used and is immediately replaced by the second set of new keys. Similar problem occurs when a Mobile Network moves off from the area. The remaining of this paper 
is structured as follows. Section II provides the background material of Logical Key Hierarchy (LKH) and group key management architecture. Constraints and assumption are covered in this section too. In Section III, we describe our two group key management schemes. We present our analytical comparison and simulation results in Section IV and V respectively. Finally, we list our conclusion in Section VI.

\section{BACKGROUND}

\section{A. Logical Key Hierarchy $(L K H)$}

For a a balanced key tree $[9,10,13]$ with outdegree, $k$, the $\mathrm{GC}$ stores all $(k N-1) /(k-1)$ keys while each member needs to store $\log _{k} N+1$ keys, where $N$ is the total number of group members. A key tree is considered balanced when the difference between any two member nodes to the root is not more than one [26]. For example, in Figure 1, member U1 knows keys $\mathrm{K} 1, \mathrm{~K} 2$ and $\mathrm{K} 5$ while member U7 knows keys $\mathrm{K} 1$, $\mathrm{K} 4$ and K11. In this example, $\mathrm{K} 1$ is the Traffic Encryption Key (TEK) which is used to encrypt the multicast data, $\mathrm{K} 2$ to $\mathrm{K} 4$ are the Key Encryption Keys (KEKs) for rekeying purposes and $\mathrm{K} 5$ to $\mathrm{K} 13$ are the individual keys associated with the group members on the leaf nodes.

When a member is removed from the group, the GC must change all the keys in the path from this member's leaf node to the root to achieve forward secrecy. All the other group members that remain in the multicast group must update their keys accordingly, namely change the keys in the intersection between the path from their leaf nodes to the root and the path from the removed member's leaf node to the root. In particular, this means that every remaining member will learn the new TEK. The rekeying cost is $k \log _{k} N-1$ keys when the key tree is balanced. For example, suppose member U9 is departing, all the keys he stores ( $\mathrm{K} 1$ and $\mathrm{K} 4$ ), except his individual key $\mathrm{K} 13$, must be changed. The GC needs to encrypt the new K4, K4', with $\mathrm{K} 11$ and $\mathrm{K} 12$ for $\mathrm{U} 7$ and $\mathrm{U} 8$. Then it encrypt $\mathrm{K} 1$ ' with $\mathrm{K} 2$, $\mathrm{K} 3$ and $\mathrm{K} 4$ ' for all group members.

If backward secrecy is required, then a join operation is similar to a remove operation in which the keys that the joining member receives must be different from the keys previously used in the group. When the key tree is balanced, the rekeying cost is $2 \log _{k} N$ keys. Suppose member U9 is joining the group, the GC first encrypts $\mathrm{K} 4$ ' with $\mathrm{K} 4$ for $\mathrm{U} 7$ and U8. Then it encrypts K1' with K1 for U1 to U8. Finally, it encrypts K1' and K4' with the individual key of U9 for U9.

\section{B. Group key Management Architecture}

Although the rekeying cost in LKH increases linearly with logarithm of the group size for a join or depart request, significant communication costs can be introduced if the group size is large with frequent join or depart requests. Furthermore, it suffers from "1 affect $N$ " problem [15], where action of one member, i.e. join or depart request, affects all the other group members.

One approach to achieve scalability is to partition the group members into a number of smaller areas to distribute the processing workload $[19,20]$. Figure 2 illustrates the group key management architecture; all members belong to a domain, denoted by the collection of cells, managed by a Domain Group Controller (DGC). The domain is divided into several areas, each managed by an Area Group Controller (AGC) within it. We partition the members on physical basis; therefore the member movement within an area does not require any rekeying. Rekeying is only needed when a member transfers between areas, joins or departs in one area.

During the multicast session, the DGC is responsible for generating the group key and distributes it to all AGCs. Each AGC can use any scalable algorithms such as LKH to distribute the group key to the members. It is important to note that the root of the key tree is now become a KEK as well. In order not to overload the DGC, the registration and deregistration of the members are carried out by the AGC too.

\section{Constraints and Assumption}

In this work, we focus on strict security. This means no member can decrypt the multicast data unless he/she is currently a member of the multicast group. Specifically, the following constraints are enforced:

- Backward Secrecy: When a member joins the multicast group, the group key must be changed to ensure that the new member cannot decode any previously transmitted multicast data.

- Forward Secrecy: When a member departs the multicast group, the group key must be changed to ensure that the departing member cannot decode future data transmissions.

For both backward and forward secrecy, the group key algorithm must halt the multicast data transmission until all the remaining members receive the group key securely. There are several assumptions we have made:

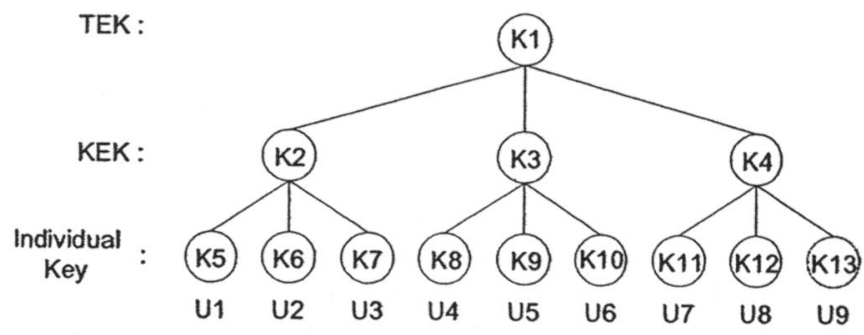

Figure 1. Logical key tree

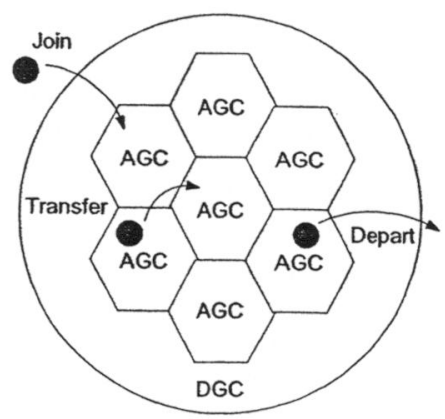

Figure 2. Group key management architecture 
- Reliable Delivery of rekeying messages: We assume that all rekeying messages are reliably received by the group members.

- Group Membership Policy Decisions: We assume that the GCs possess sufficient information to determine which members are allowed to participate.

\section{NETWORK MOBILITY REKEYING SCHEMES}

In order to reduce network resources and computation powers needed by the AGC and group members in one area when a Mobile Network moves in or out of an area, we propose two group key management schemes which treat the mobile members in a Mobile Network as a whole. Both schemes can adopt any scalable key tree algorithms such as LKH. Our schemes are different from those proposed in $[16,18,21,22$, 27] because we are more interested in mobility over large area.

\section{A. Delayed Network Mobility Rekeying Scheme (DNMRS)}

In our first scheme, Delayed Network Mobility Rekeying Scheme (DNMRS), we delay the rekeying operation by a short interval when a mobile member transfers between areas while still maintaining both forward and backward secrecy. In addition, we also partition the group members into two key trees, one for static members and one for mobile members. By separating them, we can minimize the computation powers at the static member side when a mobile member joins or departs the area and via versa.

When a static or mobile member wishes to join or depart the multicast group, the $\mathrm{AGC}$ in the area, $\mathrm{AGC} i$, will rekey the group immediately as described in section II. In addition, the AGC $i$ will inform the DGC about it and the DGC will generate a new group key and multicast it to all the AGC. In situations where a mobile member transfers between areas, the mobile member will initiate a transfer by notifying the AGC in the old area, $\mathrm{AGCi}$, and the $\mathrm{AGC}$ in the new area, AGCj. Both $\mathrm{AGC} i$ and $A G C j$ do not perform rekeying immediately; instead they wait for a short interval before rekeying the group in their area. This is because if the mobile members are traveling in a Mobile Network, all the mobile members will start to de-register with AGC $i$ and register with AGC $j$ when the Mobile Network is going to move into a new area. The interval between each joining or departing request is usually very short. It is important to note that delaying the rekeying in both areas does not affect the latency in the multicast data. This is because there is no change in the group membership, which means that the group key does not need to be changed. In other words, the DGC does not need to be notified.

If we assume that the mobile member is trusted within the multicast group, then the AGCi does not need to perform rekeying when a mobile member transfers from one area to another. Instead, the AGCi keeps a list of the mobile member that performs such transfer. This list is cleared whenever the AGCi performs a rekeying. Allowing a mobile member to have more than one set of valid keys while he/she stays in the multicast group does not compromise the requirement stated in Section II, as long as all the keys he/she possesses are updated when he/she finally departs from the multicast group. DNMRS is different compared to batch rekeying $[25,27,28]$, which trade-off security for performance, because the AGC will only delay rekeying if the mobile member is transferring between areas. Furthermore, we like to emphasize that batch rekeying does not fulfil our requirement.

As there might be several Mobile Networks in an area, additional information needed by the AGC $i$ is the default router of the mobile members. This allows AGC $i$ to place all mobile members in the mobile network together to minimize the rekeying costs if LKH is used. As shown in Figure 3, S1 to S8 are the static members and M1 to M6 are the mobile members. In the mobile member key tree, the $\mathrm{AGC} i$ groups the members in same Mobile Network together. In this case, assume Mobile Network 1 moves out from the area, no rekeying is needed since $\mathrm{K} 16$ is not needed anymore. However, if the related mobile members are not properly placed together, rekeying might be needed.

\section{B. Mobile Group Controller Scheme(MGCS)}

For our second scheme, Mobile Group Controller Scheme (MGCS), we introduce another trusted entity, known as Mobile Group Controller (MGC). A MGC is deployed on the Mobile Network and it plays a similar role as an AGC. The main difference between them is a MGC is mobile. In this case, the registration and de-registration of the mobile members in the Mobile Network are handled by the MGC. As for the static members and individual mobile members, they will register or de-register with the AGC in the area. Unlike DNMRS and other existing algorithms, a mobile member only needs to register and de-register once with the $\mathrm{MGC}$ as long as he/she stays inside the Mobile Network. Transferring between areas does not require the mobile members in the Mobile Network to de-register with the $\mathrm{AGC} i$ and re-register with the $\mathrm{AGC} j$.

In MGCS, the MGC can receive the group key either from the DGC or the AGC in that area. Since it is possible for the Mobile Network to experience loss of service during movement therefore it is preferably for the MGC to obtain the group key from the AGC in that area. In addition, the MGC should confirm with the present AGC that the group key has not been changed when the Mobile Network moves into a new area. Compared with existing schemes, this scheme reduces the latency needed for rekeying to almost none since there is no change in the group membership. Furthermore, no computation is needed by the AGC and the group members as there is no change in the group membership when a Mobile Network moves in or out of an area.
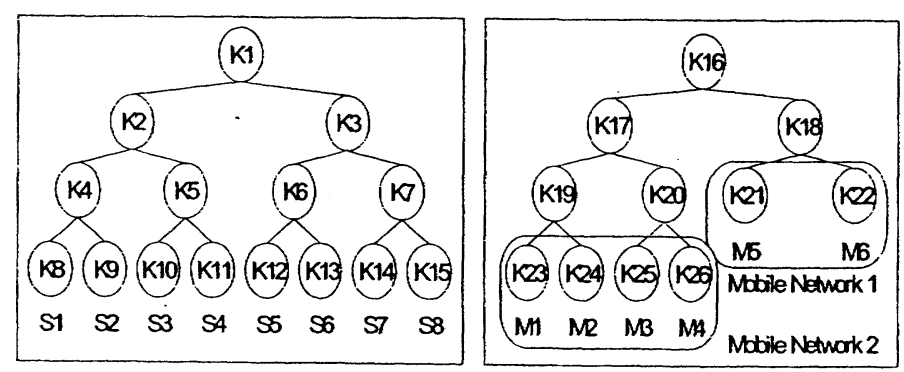

Figure 3. Key tree for static and mobile members 


\section{ANALYTICAL COMPARISON}

In this section, we focus on three performance metrics: (i) group member key storage, (ii) GC key storage and (iii) rekeying costs for the various join and depart request in one area. For simplicity, we adopt LKH in our two schemes but the other scalable algorithms will yield similar performance too. We assume that there is only one Mobile Network in the area, with a total number of $M$ mobile members and the total number of static members is $N_{0}$.

Table I examines the key storage needed by the AGC, MGC and group members. Generally, all three AGCs have similar key storage. The only difference is that the MGCS requires another trusted entity, $\mathrm{MGC}$, to handle the mobile members in the Mobile Network.. As for the group members, both static and mobile members in LKH have similar key storage as they are placed in a single key tree. In the case of DNMRS and MGCS, the static members and the mobile members are in separate key tree, which leads to different key storage between them. This is advantageous for mobile members as mobile members might have limited storage.

Table II examines the rekeying costs for the various join or depart event. It can be seen that both static and mobile members in LKH have similar rekeying costs for each individual event as they are placed in the same key tree. As for

TABLE I. KEY STORAGE FOR AGC, MGC AND GROUP MEMBERS

\begin{tabular}{|c|c|c|c|}
\hline & LKH & DNMRS & MGCS \\
\hline Key storage for AGC & $\frac{k\left(N_{0}+M\right)-1}{k-1}+1$ & $\frac{k\left(N_{0}+M\right)-2}{k-1}+1$ & $\frac{k N_{0}-1}{k-1}+1$ \\
\hline Key storage for static member & $\left\lceil\log _{k}\left(N_{0}+M\right)\right\rceil+2$ & $\left\lceil\log _{k} N_{0}\right\rceil+2$ & $\left\lceil\log _{k} N_{0}\right\rceil+2$ \\
\hline Key storage for mobile member & $\left\lceil\log _{k}\left(N_{0}+M\right)\right\rceil+2$ & $\left\lceil\log _{k} M\right\rceil+2$ & $\left\lceil\log _{k} M\right\rceil+2$ \\
\hline Key storage for MGC & - & - & $k M-1$ \\
\hline
\end{tabular}

TABLE 1I. REKEYING COSTS FOR GROUP MEMBERS FOR VARIOUS JOIN OR DEPART EVENT

\begin{tabular}{|c|c|c|c|c|}
\hline & & LKH & DNMRS & MGCS \\
\hline \multirow{2}{*}{$\begin{array}{l}\text { Static member } \\
\text { joins the area }\end{array}$} & $\begin{array}{l}\text { Rekeying costs for } \\
\text { static member }\end{array}$ & $2\left\lceil\log _{k}\left(N_{0}+M\right)\right\rceil+1$ & $2\left\lceil\log _{k} N_{0}\right\rceil+1$ & $2\left\lceil\log _{k} N_{0}\right\rceil+1$ \\
\hline & $\begin{array}{l}\text { Rekeying costs for } \\
\text { mobile member }\end{array}$ & $2\left\lceil\log _{k}\left(N_{0}+M\right)\right\rceil+1$ & 1 & 1 \\
\hline \multirow{2}{*}{$\begin{array}{l}\text { Static member } \\
\text { departs the area }\end{array}$} & $\begin{array}{l}\text { Rekeying costs for } \\
\text { static member }\end{array}$ & $k\left\lceil\log _{k}\left(N_{0}+M\right)\right\rceil$ & $k\left\lceil\log _{k} N_{0}\right\rceil$ & $k\left\lceil\log _{k} N_{0}\right\rceil$ \\
\hline & $\begin{array}{l}\text { Rekeying costs for } \\
\text { mobile member }\end{array}$ & $k\left\lceil\log _{k}\left(N_{0}+M\right)\right\rceil$ & 1 & 1 \\
\hline \multirow{2}{*}{$\begin{array}{l}\text { Mobile Network } \\
\text { moves into } \\
\text { the area }\end{array}$} & $\begin{array}{l}\text { Rekeying costs for } \\
\text { static member }\end{array}$ & $\sum_{m=1}^{M} 2\left\lceil\log _{k}\left(N_{0}+m\right)\right\rceil$ & - & - \\
\hline & $\begin{array}{l}\text { Rekeying costs for } \\
\text { mobile member }\end{array}$ & $\sum_{m=1}^{M} 2\left\lceil\log _{k}\left(N_{0}+m\right)\right\rceil$ & $\frac{k(M-1)}{k-1}$ & - \\
\hline \multirow{2}{*}{$\begin{array}{l}\text { Mobile Network } \\
\text { moves out of } \\
\text { the area }\end{array}$} & $\begin{array}{l}\text { Rekeying costs for } \\
\text { static member }\end{array}$ & $\sum_{m=1}^{M} k\left\lceil\log _{k}\left(N_{0}+m\right)\right\rceil-1$ & - & - \\
\hline & $\begin{array}{c}\text { Rekeying costs for } \\
\text { mobile member }\end{array}$ & $\sum_{m=1}^{M} k\left\lceil\log _{k}\left(N_{0}+m\right)\right\rceil-1$ & - & - \\
\hline
\end{tabular}


DNMRS and MGCS, the static and mobile members are placed on separate key trees, therefore the join or depart request of a static member does not affect the key tree of the mobile members. Regardless of whether the Mobile Network moves in or out of an area, the static and mobile members in MGCS do not need any rekeying if there is no change in the group membership. In the case of LKH, when the Mobile Network moves in or out of an area, the rekeying costs can overwhelm the AGC and the static members as each mobile member starts to de-register with the old AGC and register with the new AGC. This is because LKH did not anticipate the mobile members in the Mobile Network, therefore it performs a rekeying each time a mobile member joins or departs the area, resulting in a waste of network resources and computation power.

\section{COMPARISON USING SUMUlation}

In this section, we use simulation to get a better understanding on the rekeying costs for the static and mobile members when the Mobile Network moves in and out of the area. The worst case is assumed although the result might be better. For our simulation, we use a balanced binary key tree $(k=2)$. There are a total of 8192 static members in the area and we vary the total number of mobile members in the Mobile Network from 1 to 200. Similarly as in section IV, we assume that there is only one Mobile Network in the area.

Figure 4 shows the rekeying costs for the static and mobile members when the Mobile Network moves into the area. We can see that the rekeying cost for static and mobile members in LKH is very high compared to DNMRS and MGCS. This is because it did not consider mobile members in Mobile Network as a whole. In our simulation, we assume that the mobile members register and de-register within the waiting period in the DNMRS. If the mobile members in the Mobile Network register outside the waiting period, the rekeying costs will be slightly higher than the one shown below. The static members in DNMRS do not need any rekeying since there is no change in group membership. As for the mobile members in DNMRS, the rekeying cost consists of the cost to create a new key tree. In the case of MGCS, no rekeying is needed for both the static and mobile members when the Mobile Network moves into an area since there is no change in the group membership.

Figure 5 shows the rekeying costs for the static and mobile members when the Mobile Network moves out of the area. Similarly, LKH incurs huge rekeying costs due to the lack of anticipation of the mobile members in the Mobile Network. A rekeying is performed every time a mobile member de-registers with the old AGC. As for DNMRS and MGCS, rekeying is not needed. This is because the mobile members are placed on separate key tree; therefore the key tree of the static members is not affected when the mobile members transfer to another area.

\section{CONCLUSION}

In this paper, we presented two group key management schemes, which consider mobile members in Mobile Network as a whole. Doing so can reduce the rekeying costs by a significant amount when the Mobile Network moves in or out of the area compared to existing algorithms. Proper placement

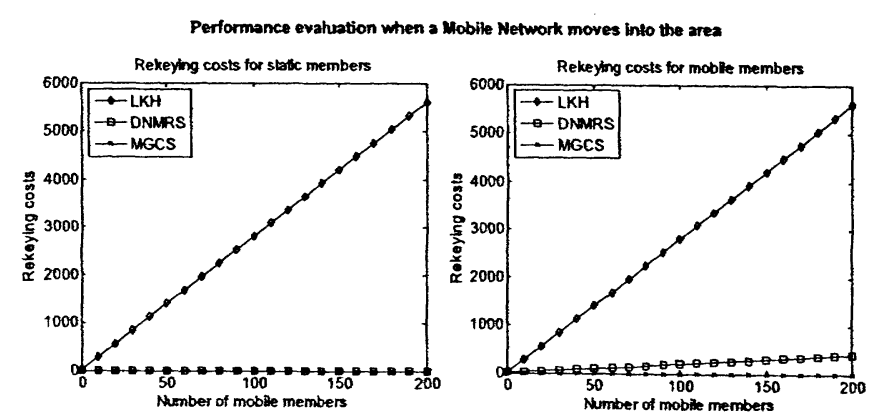

Figure 4. Rekeying costs when a Mobile Network moves into the area
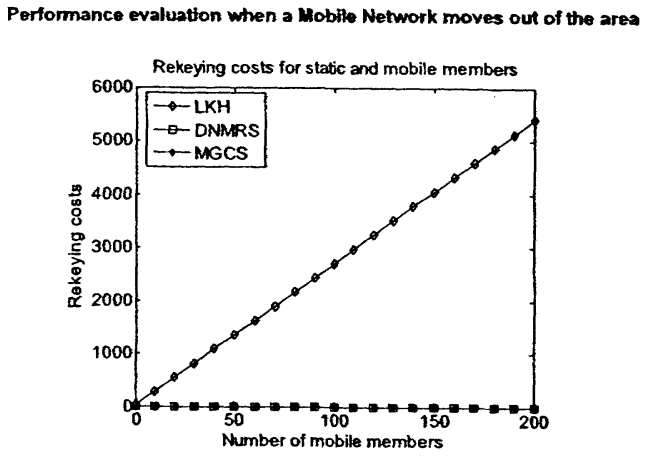

Figure 5. Rekeying costs when a Mobile Network moves out of the area

of the mobile members in the key tree is also taken into consideration in order to minimize the rekeying costs.

The first scheme, DNMRS, delays the rekeying operation when a mobile wishes to transfer between areas. In addition, the group members are partitioned into two key trees: one for static members and one for mobile members. This approach has several advantages. First, the key storage of the mobile members is minimized and this benefits mobile devices with limited storage. Second, the join or depart event of a static member does not affect the mobile members and similarly the join and depart event of the mobile member does not affect the static members since both of them are placed on separate key tree. This benefits those mobile devices with limited computation power. Reducing the number of decryptions can also help to conserve energy which leads to battery saving. Third, even though the AGC needs to wait for a short time period, which is sufficient long enough for all mobile members in the Mobile Network to send their registration or deregistration when the Mobile Network moves in or out of the area, it does not affect the latency in the multicast data since the multicast data is not halted.

The second scheme, MGCS, introduces another trusted entity, MGC, which is responsible for all the mobile members in the Mobile Network. All mobile members in the Mobile Network need to register or de-register with the MGC only once when they want to join or depart from the multicast session. Similarly as the DNMRS, the joining and departing of a static member does not affect the mobile members. As long as there is no change in the group membership, no rekeying cost is incurred during the movement. Compared to DNMRS, this approach is better since the mobile members do not need to 
de-register with the old AGC and register with the new AGC when the Mobile Network moves from one area to another.

\section{REFERENCES}

[1] S.E. Deering, "Host Extensions for IP Multicasting", RFC 1112, IETF, Aug 1989.

[2] S. Paul, "Multicast on the Internet and its applications", Kluwer Academic Publishers, 1998.

[3] U. Varshney, "Multicast over wireless networks", Communications of ACM, Vol. 45, Issue 12, pp. 31-37, Dec. 2002.

[4] U. Varshney, "Multicast support in mobile commerce application", IEEE Computer, Vol. 35, Issue 2, pp. 115-117, Feb 2002.

[5] N. Shi, "Mobile commerce applications", Idea Group Publishers, 2004.

[6] R. Canetti, J. Garay, G. Itkis, D. Micciancio, M. Noar, B. Pinkas, "Multicast security: A taxonomy and efficient constructions", In Proc. IEEE INFOCOM, Vol. 2. pp. 708-716, Mar. 1999.

[7] H. Harney and C. Muckerhirn, "Group key management protocol (GKMP) specification", RFC 2093.

[8] H. Harney and C. Muckenhim, "Group key management protocol (GKMP) architecture", RFC 2094.

[9] D.M. Wallner and E.J. Harder and R.C. Agee, "Key management for multicast issues and architectures", RFC 2627, Jun. 1999.

[10] C. Wong, M. Gouda and S. Lam, "Secure group communication using key graphs", IEEE/ACM Trans. Networking, Vol. 8. pp. 12-23, Feb. 2000

[11] D. Balenson, D. McGrew and A. Sherman, "Key Management for large dynamic groups: One-way function trees and amortised initialisation", Internet Draft, draft-irtf-smug-groupkeymgmt-oft-00.txt. Aug. 2000.

[12] M. Valdvogel, G. Caronni, D. Sun, N. Weiler and B. Plattner, "The versakey frameworks: versatile group key management", IEEE JSAC (Special Issue on Middleware), Vol. 17, No. 9, pp. 1614-1631, Sept 1999.

[13] M.P. Howarth, S. lyengar, Z. Sun, H. Cruickshank, "Dynamics of key management in secure satellite multicast", IEEE JSAC, Feb 2004.

[14] A. Ballardie, "Scalable multicast key distribution", RFC 1949, 1996.

[15] S. Mittra, "lolus: A framework for scalable secure multicasting", In Proc. ACM SIGCOMM, Vol. 27, pp. 277-288, Sept. 1997.

[16] B. DeCleene, L. Dondeti, S. Griffin, T. Hardjono, D. Kiwior, J. Kurose, D. Towsley, S. Vasudevan, C. Zhang, "Secure group communication for wireless networks", In Proc. MILCOM, Oct. 2001.
[17] A. Perrig, "Efficient collaborative key management protocol for secure autonomous group communication", In Proc. International Workshop on Cryptographic Techniques and E. Commerce (CrypTEC), pp. 192-202, 1999.

[18] C. Zhang, B. DeCleene, J. Kurose, D. Towsley, "Comparison of interarea rekeying algorithms for secure wireless group communications", In Proc. Performance Evaluation, Vol. 49, pp. 1-20, Sept. 2002.

[19] T. Hardjono, B. Cain, I. Monga, "Inter-domain group key management protocol", Internet Draft, draft-irtf-smug-intragkm-00.txt, Sept. 2000.

[20] M. Baugher, R. Canetti, L. Dondeti, F. Lindholm, "Group key management architecture", Internet Draft, draft-msec-gkmarch-08.txt, Jun. 2004.

[21] L. Dondeti, B. DeCleene, S.P. Griffin, T. Hardjono, J. Kurose, D. Townsley, C. Zhang, S. Vasudevan, "Group key management in wireless and mobile environments", Internet Draft, draft-dondeti-irtf-smug-gkmmobility-00.txt, Jul. 2001.

[22] S.P. Griffin, B. DeCleene, L. Dondeti, R.M. Flynn, D.Kiwior, A. Olbert, "Hierarchical key management for mobile multicast members", Technical Report, Northrop Grumman Information Technology, 2002.

[23] H.Y. Lach, C. Janneteau and A. Petrscu, "Network mobility in beyond3G systems", IEEE Communication Magazine, Jul. 2003.

[24] T. Ernst, "Network mobility support goals and requirements", Internet Draft, draft-ietf-nemo-requirement-03.txt, Oct. 2004.

[25] X.S. Li, Y.R. Yang, M. Gouda, S. Lam, "Batch rekeying for secure group communications", In Proc. $10^{\text {th }}$ International WWW Conference, pp. 525-534, May 2001.

[26] M.J. Moyer, J.R. Rao, P. Rohatgi, "Maintaining balanced key trees for secure multicast", Internet Draft, draft-irtf-smug-key-tree-balance-00.txt, Jun. 1999.

[27] Y. Sun, W. Trappe, K.J.R. Liu, "A Scalable Multicast Key Management Scheme for Hetergenous Wireless Networks", IEEE/ACM Trans. Networking, Vol. 12, pp. 653-666, Aug. 2004.

[28] S. Setia, S. Koussih, S. Jajodia, "Kronos: A scalable group rekeying approach for secure multicast", In Proc. IEEE Symposium on Security and Privacy, 2000.

[29] X.B. Zhang, S. Lam, D.Y. Lee, Y.R. Yang, "Protocol design for scalable and reliable group rekeying", IEEE/ACM Trans. Networking, Vol. 11, pp. 908-922, Dec 2003. 